\title{
Radiation-induced modifications of the tumor microenvironment promote metastasis
}

\author{
Curzio Rüegg ${ }^{1,2,3}$, Yan Monnier ${ }^{1,3}$, François Kuonen ${ }^{1,3}$, Natsuko Imaizumi ${ }^{1,3}$ \\ ${ }^{1}$ Division of Experimental Oncology, Multidisciplinary Oncology Center (CePO), University Medical Center (CHUV), \\ University of Lausanne (UNIL), Lausanne, Switzerland \\ ${ }^{2}$ Laboratory of tumor angiogenesis and metastasis, Pathology, Department of Medicine, Faculty of Science, University of \\ Fribourg (UNIFR), Fribourg, Switzerland \\ <curzio.ruegg@unifr.ch> \\ ${ }^{3}$ National Center for Competence in Research (NCCR), Molecular Oncology, Swiss Institute for Experimental Cancer \\ Research (ISREC)-École Polytechnique Fédérale de Lausanne (EPFL), Lausanne, Switzerland
}

Abstract. Radiotherapy is successfully used to treat cancer. Emerging evidence, however, indicates that recurrences after radiotherapy are associated with increased local invasion, metastatic spreading and poor prognosis. Radiation-induced modifications of the tumor microenvironment have been proposed to contribute to increased aggressive tumor behavior, an effect also referred to as tumor bed effect, but the putative mechanisms involved have remained largely elusive. We have recently demonstrated that irradiation of the prospective tumor stroma impairs de novo angiogenesis through sustained inhibition of proliferation, migration and sprouting of endothelial cells. Experimental tumors growing within a pre-irradiated field have reduced tumor angiogenesis and tumor growth, increased hypoxia, necrosis, local invasion and distant

\section{Introduction}

About half of cancer patients are treated by radiotherapy for curative-intent, adjuvant or palliative purposes during the course of their disease [1]. It is generally assumed that the anti-tumor effect of radiotherapy depends on tumor cell DNA-damage leading to p53-mediated apoptosis, mitotic cell death or senescence-like irreversible growth arrest [2]. Although radiotherapy is supposed to target tumor cells, it also hits the tumor microenvironment. Radiation-induced modifications of the tumor microenvironment, including both cellular and extracellular elements, may further contribute to the therapeutic effect of radiotherapy [3]. For example, irradiation of established tumors kills angiogenic endothelial cells, resulting in the disruption of the tumor vasculature and enhanced metastasis. Mechanisms of progression involve adaptation of tumor cells to local hypoxic conditions as well as selection of cells with invasive and metastatic capacities. The matricellular protein CYR61 and integrin $\alpha \mathrm{V} \beta 5$ emerged as molecules that cooperate to mediate lung metastasis. Cilengitide, a small molecular inhibitor of $\alpha \mathrm{V}$ integrins prevented lung metastasis formation. These results represent a conceptual advance to the understanding of the tumor bed effect and indicate that $\alpha \mathrm{V}$ integrin inhibition might be a potential therapeutic approach for preventing metastasis in patients at risk for post-radiation recurrences. $\triangle$

Keywords: radiotherapy, angiogenesis, integrins, $\alpha \mathrm{V} \beta 5$, CYR61, TGF- $\beta$, tumor bed effect, invasion, metastasis

anti-tumor effect [4]. However, growing clinical and experimental evidence indicates that the irradiated tumor microenvironment or irradiated normal tissue may exert potential tumor-promoting effects. For instance, adjuvant radiotherapy significantly improves local tumor control in patients undergoing conservative surgery, including breast and head and neck cancers, but tumor recurrences within a pre-irradiated field is often associated with higher risk of metastasis and poor prognosis compared to recurrences outside the irradiated area [5-9]. Experimentally, tumors growing within a previously irradiated bed tend to be more metastatic. This effect has been previously termed tumor bed effect $[10,11]$. A recent study analyzing retrospective datasets, however, reported that recurrences after postmastectomy radiation therapy are not associated with 
more aggressive local behaviors or reduced survival compared to patients recurring after mastectomy only [12]. Thus, at this point this question remains open and controversial. In the light of these considerations, it is important to realize that only little is known about the cellular and molecular mechanisms mediating the increased metastatic capacity of experimental tumors developing within a pre-irradiated field [13-15].

\section{Effect of radiotherapy on tumor vessels}

Garcia-Barros et al., originally reported that administration of high doses (i.e. 15 Grey [GY]) of ionizing radiations to tumors induced ceramide-mediated apoptosis of endothelial cells of the tumor vasculature, causing tumor vessel disruption and delayed tumor growth. Prevention of ceramide-mediated endothelial cell apoptosis attenuated the anti-tumor effect of radiotherapy, thereby demonstrating that tumorassociated endothelial cells represent a critical target of radiotherapy determining its therapeutic efficacy [4]. Proliferating microvascular endothelial cells undergo two waves of death after exposure to single high doses of radiation: an early pre-mitotic membrane-dependent apoptosis, which requires ceramide generation, and a delayed mitotic death, which depends on DNA damage. Sphingosine-1-phosphate (S1P), a ceramide antagonist, protects endothelial cells only from membrane-dependent apoptosis but not from DNA damage-induced mitotic death [16]. Consistent with a cytotoxic effect of radiotherapy on angiogenic vessels, concomitant administration of anti-angiogenic drugs impinging on endothelial cell survival pathways, such as anti-vascular endotehlial growth factor (VEGF) antibodies (e.g. bevacizumab) [17], VEGF receptor tyrosine kinase inhibitors (e.g. AG-013736) [18] or mTOR inhibitors (e.g. RAD001) [19], sensitize angiogenic endothelial cells to ionizing radiation-induced death, thereby enhancing tumor vascular damage induced by radiotherapy and improving therapeutic response [20].

\section{Effect of ionizing radiation on normal, quiescent vessels}

In addition to angiogenic tumor vessels, high-dose ionizing radiation also affects quiescent vessels in healthy tissues in a dose- and time-dependent manner. Microvessels (capillaries and sinusoids) are most sensitive to ionizing radiation, displaying damage of endothelial cells, telangectasia, rupture and thrombosis. Irradiated medium-size vessels show neointimal proliferation, fibrinoid necrosis, thrombosis and acute arteritis. Large vessels are less sensitive to radiation, although arterial thrombosis and atheromatosis were reported [21]. These radiation-induced vascular modifications are associated with increased risk of serious complications in radiotherapy-treated patients, including arterial occlusions, heart attacks and stroke [22-25]. Radiation-induced endothelial lesions have also been proposed to be responsible for tissue damages and dysfunctions, in particular, in initiating the lethal gastrointestinal syndrome, which occurs upon exposure of the abdomen to radiation. It consists of diarrhea, dehydration, bacterial infection, possibly leading to septic shock and death [26]. Using mouse models, the Kolesnick laboratory has shown that microvascular endothelial apoptosis is the primary lesion leading to intestinal epithelial stem cell dysfunction, crypt damage, organ failure, and death, following high-dose abdominal irradiation [27]. Administration of fibroblast growth factor-2 (FGF-2) or genetic deletion of the acid sphingomyelinase gene protected intestinal endothelial cells against radiation-induced death and mice against radiation-induced gastrointestinal syndrome [27, 28]. This proposed role of endothelial cell apoptosis in the initiation of the radiation-induced gastrointestinal syndrome, however, was recently challenged by the finding that selective deletion of $p 53$ from the gastrointestinal epithelium, but not from endothelial cells, sensitized irradiated mice to the gastrointestinal syndrome [29].

\section{Ionizing radiation inhibits angiogenesis through the transforming growth factor beta (TGF $\beta$ ) RI/activin receptor-like kinase-5 pathway}

It has been long suspected that exposure of healthy tissues to ionizing radiation may inhibit a subsequent angiogenic response. Endothelial dysfunctions and impaired angiogenesis are thought to contribute to late tissue damages observed after radiotherapy, including poor wound healing [30]. In spite of its clinical relevance, whether irradiation of quiescent vasculature impinges on subsequent (de novo) angiogenesis and the putative mechanisms involved have 
not been thoroughly investigated. We have recently studied the effects of ionizing radiation on angiogenesis through in vivo, ex vivo and in vitro experiments, in combination with genetic and pharmacological interventions. We have observed that high doses of ionizing radiation (8-20 Gy) locally suppressed FGF2- and VEGF-induced angiogenesis in the Matrigel in vivo assay without disrupting preexisting dermal vessels. The effect is local, since angiogenesis was normal in Matrigel plugs implanted contralaterally to the irradiation site. Irradiation of quiescent mouse aorta in vivo or ex vivo suppressed endothelial cell sprouting in response to VEGF in the ex vivo aortic ring sprouting assay. Irradiation of endothelial cells in vitro inhibited sprouting, migration, proliferation, and caused premature senescence and enhanced matrix adhesion, without increasing cell death [31]. Taken together these results indicate that endothelial cells are direct targets of ionizing radiations (figure 1) .

Two pathways are known to induce cell cycle arrest and senescence: the P16-Rb and P53-P21 pathways. Radiation activates P53 mostly through the induction of DNA double-strand breaks [32]. Irradiation of confluent endothelial cells induced DNA breaks, which were largely repaired within twenty-four hours after irradiation. Western blotting for P53, P21 and P16 proteins revealed a rapid increased of P53 levels followed by an increase in P21 levels. P16 levels remained unchanged, suggesting a role for P53 or $\mathrm{P} 21$ in radiation-induced senescence and proliferation arrest. However, $p 53$ or $p 21$ deficiency in murine endothelial cells in vivo, or P21 silencing in cultured human endothelial cells in vitro did not prevent radiation-induced inhibition of sprouting or proliferation [31]. Radiation was reported to activate TGF- $\beta$ and TGF- $\beta$ signaling in vitro and in vivo $[33,34]$. We therefore tested whether TGF- $\beta$ signaling was activated in our model by monitoring mothers against decapentaplegic (SMAD-2) phosphorylation. Radiation induced SMAD-2 phopsphorylation and downstream signaling in endothelial cells. Pharmacological inhibition of the TGF- $\beta$ receptor type I ALK5 with the kinase inhibitor SB431542 rescued radiation-induced cell sprouting and migration defects in vitro and restored angiogenesis in irradiated mice in vivo [31]. These results clearly demonstrate that irradiation of quiescent endothelial cells suppresses subsequent de novo angiogenesis and that TGF- $\beta$ RI/ALK5 is a critical mediator of this effect.

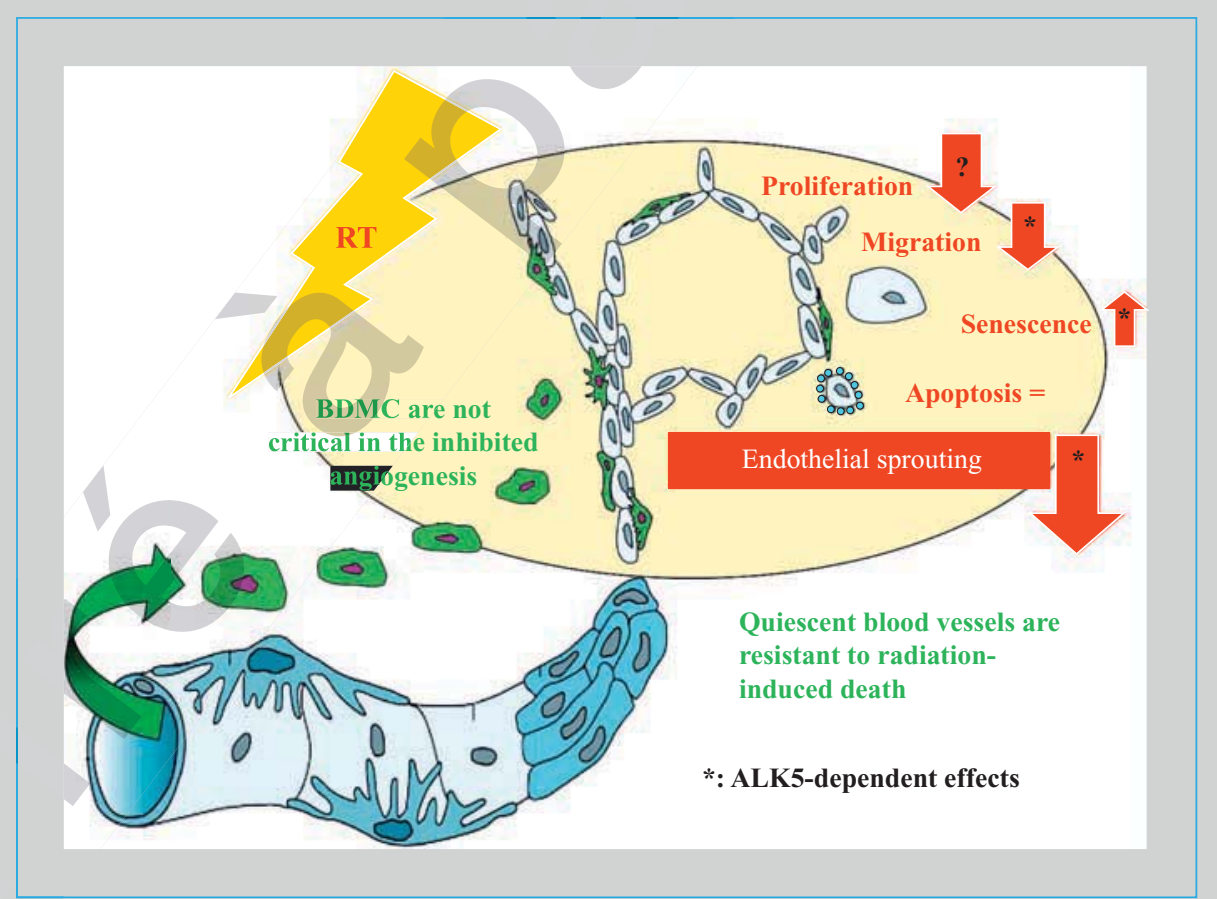

Figure 1. Working model of radiation-induced inhibition of sprouting angiogenesis.

Ionizing radiation impairs proliferation, migration, and sprouting and promotes senescence but not apoptosis, of quiescent endothelial cells. Leukocyte recruitment into irradiated beds is decreased by irradiation, but not critical in determining impaired angiogenesis. *: activin receptor-like kinase-5-dependent effects reversed by a activin receptor-like kinase-5 inhibitor; ?: the mechanism of action remains unknown; RT: radiotherapy. 
In these experiments, we could not determine the mechanisms inhibiting endothelial cell proliferation and causing senescence. This is likely to reflect the complexity and redundancy of the mechanisms controlling such vital cellular functions as well as the multiplicity of signaling events elicited by ionizing radiations. Unraveling the mechanism of inhibited proliferation will require significant further work and may profit from a system biology approach [35]. The demonstration that inhibition of de novo angiogenesis is a direct effect of high-doses ionizing radiations on endothelial cells suggests that curative-intent and adjuvant radiotherapy might act, at least in part, by inhibition angiogenesis.

\section{Tumors growing in pre-irradiated fields have reduced microvascular density, are hypoxic and become invasive and metastatic}

How do tumors that grow within a pre-irradiated field with deficient angiogenic capacity evolve? We have addressed this question by implanting SCCVII (murine squamous cell carcinoma) and HCT116 (human colorectal cancer) tumor cells subcutaneously into immunosuppressed mice locally pre-irradiated with 20 Gy. Such tumors had decreased microvascular density, thus confirming the observations made in the Matrigel plug assays. Tumors were smaller and their margins were highly invasive compared to tumors implanted in non-irradiated mice [36]. They also had increase hypoxia (detected by hypoxiprobe) and necrosis. We also observed a significant increase in lung metastasis formation in mice bearing SCCVII or HCT116 tumors growing within pre-irradiated skin compared to non-irradiated tumor-bearing mice. These models had the limitation of being subcutaneous and possibly not reflecting a clinically relevant condition. We therefore developed orthotopic breast cancer transplantation models in which murine $4 \mathrm{~T} 1$ or human MDA-MB-231 breast cancer-derived lines are injected in pre-irradiated mammary fat pads of immunocompetent (BALB/c) mice or immunodeficient (Swiss nu/nu) mice, respectively. In both models the prospective irradiation of the mammary gland inhibited primary tumor growth, increased local invasion, enhanced lymphatic spreading to ipsilateral lymph nodes and promoted hematogenous metastases to lungs and liver. Both $4 \mathrm{~T} 1$ and MDA-MB-231 tumors growing in preirradi- ated mammary glands had decreased microvascular density, consistent with radiation-induced inhibition of angiogenesis, and were more hypoxic and necrotic. In these models we observed increased tumor infiltration by $\mathrm{CD} 11 \mathrm{~b}^{+}$myelo-monocytic cells, especially at the invasive margins, in irradiated mice compared to nonirradiated ones (F. Kuonen, manuscript in preparation). These results demonstrated that pre-irradiation of the tumor bed reduces local tumor growth, likely due to suppressed angiogenesis and metabolic starvation, but at the same time enhances local invasion and metastatic spreading to lymph nodes and distant organs. They also pointed to the likely involvement of hypoxia in promoting metastasis. Others have previously shown that experimental tumors growing in pre-irradiated tissues and human tumors locally recurring after radiotherapy have an increased fraction of hypoxic cells [37-39], and that experimental tumors with a high hypoxic fraction do metastasize more frequently compared to tumors with low a hypoxic fraction [40, 41]. Rofstad et al. showed that enhanced invasion and metastasis observed in tumors growing within a pre-irradiated bed involves hypoxia-mediated up-regulation of metastasispromoting gene products, in particular the receptor for the urokinase-type plasminogen activator receptor (UPAR) [14]. These observations are of clinical relevance, since tumoral hypoxia is associated with local tumor invasion, metastasis formation and shorter disease-free survival in a number of human tumors, including head and neck cancer [42], cervical cancer [43] and soft tissue sarcoma [44]. Taken together, these results suggested that primary tumors recurring after radiation treatment show aggressive behavior in association with increased environmental hypoxia (figure 2).

\section{Hypoxia selects invasive and metastatic tumor cells}

The transcription factor hypoxia inducible factor-1 (HIF-1) mediates adaptive responses to hypoxia and activates the transcription of genes encoding for proteins involved in angiogenesis, glucose metabolism, intracellular $\mathrm{pH}$ regulation, cell proliferation/survival, cell migration and invasion $[45,46]$. Therefore, activation of a metastatic program by HIF-1 appeared as an attractive mechanism by which tumor cells growing into a pre-irradiated hypoxic microenvironment invade and metastasize. To test for a role of HIF in promoting metastasis in our model, we examined the metastatic 


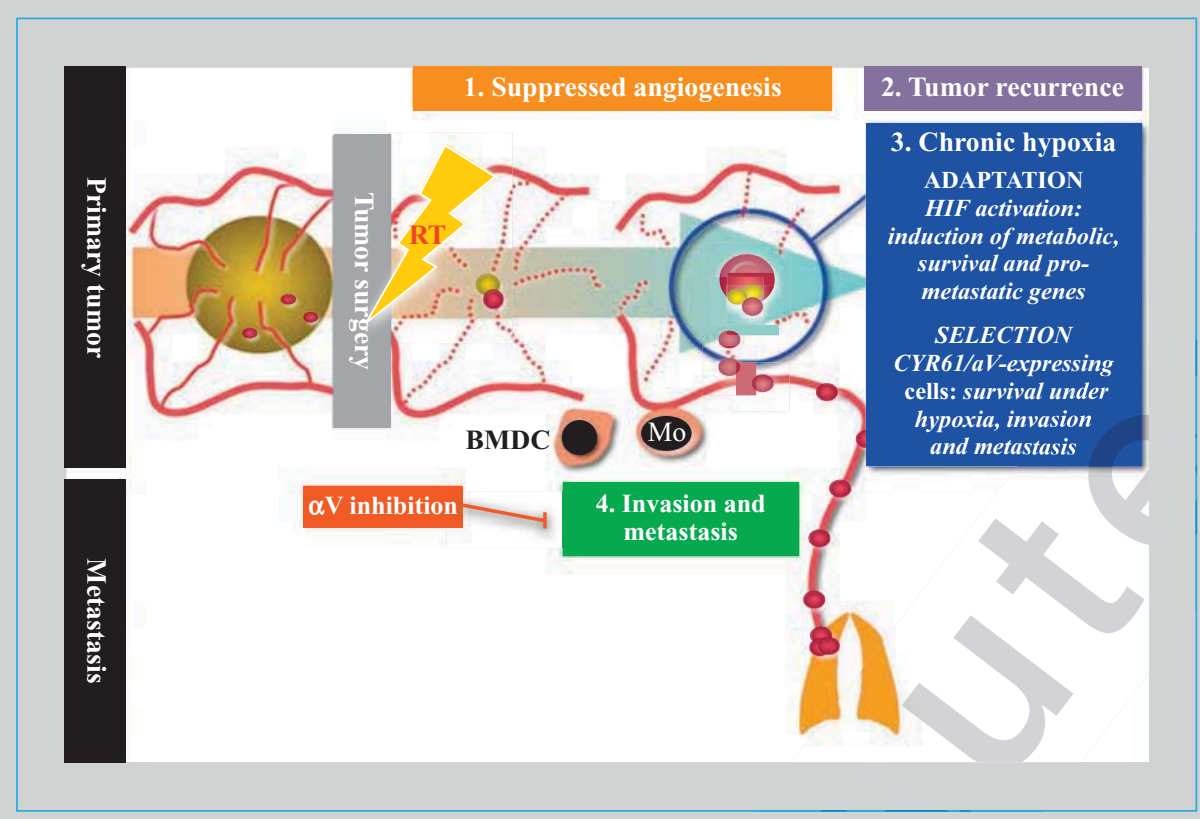

Figure 2. Working model of tumor metastasis formation after tumor bed irradiation.

The tumor itself, or its bed and the surrounding tissue after surgery are irradiated for curative intent or adjuvant treatment purposes. 1. De novo angiogenesis is impaired in irradiated tissue. 2. Tumors recurring within this angiogenesis-deficient bed develop areas of hypoxia. 3. Hypoxia induces an adaptive response, for example via HIF and also selects tumor cells resistant to hypoxia-induced death. 4. CYR61/ $\alpha \mathrm{V}$ integrin-expressing cells are resistant to hypoxia, and possess invasive and metastatic capacities. Inhibition of $\alpha \mathrm{V}$ integrin inhibits metastasis induced by CYR61. RT: radiotherapy.

capacity of two congenic HEPA1-derived tumor cell lines with either functional or non-functional HIF [47]. Pre-irradiation of the prospective tumor bed strongly decreased the growth of both lines and HIF deficiency inhibited tumor growth on both irradiated and nonirradiated beds. Absence of HIF strongly reduced the overall metastatic ability of tumors developing on both non-irradiated and pre-irradiated bed, however, the relative increase (4-6 fold) in lung metastasis formation of tumors growing in pre-irradiated vs. non-irradiated bed remained unchanged [36]. This result suggested that additional, HIF-independent, mechanisms were involved. To test this hypothesis we derived lines from tumors grown in a pre-irradiated bed in vivo, or through repeated cycles of severe hypoxia in vitro. Derived cell lines retained invasive capacities in vitro, even when subsequently returned to normoxia, and increased metastatic capacities in vivo when injected into nonirradiated mice, while their primary tumor growth was identical [36]. All derived lines had low HIF-1 $\alpha$ protein levels under normoxia and high levels under hypoxia, thereby excluding that invasive/metastatic cell sub-populations were selected on the basis of constitutive HIF- $1 \alpha$ activation. These data demonstrate that pre-irradiated tumor bed selected for invasive and metastatic tumor cells that retained invasive and metastatic capacities upon return to normoxia. It is worth noting that the induction of a more invasive and metastatic phenotype following therapeutic induction of tumor hypoxia was subsequently observed for tumors treated with anti-angiogenic drugs. Using the Rip1 Tag2 model of multistep carcinogenesis and a glioblastoma model, Paez-Ribes et al., reported that treatment with angiogenesis inhibitors targeting the VEGF pathway caused primary tumor reduction while promoted progression to invasiveness and increased metastasis [48]. The putative mechanism involved, however, was not identified in this model. Collectively these findings suggest that increased invasion and metastasis may be a common condition in situation where tumor angiogenesis is suppressed and tumor cells endure increased metabolic stress.

\section{Cysteine-rich protein 61 and $\alpha \mathrm{V}$ integrins co-operate to mediate metastasis}

To identify genes linked to the metastatic capacity of tumor cells growing in a pre-irradiated bed, we performed gene expression studies. Ninety-two probe 
sets were found differentially expressed between lines derived from tumors growing in irradiated vs. nonirradiated beds. The cysteine-rich protein 61 (CYR61), a member of the CCN (CYR61/CTGF/NOV) family of matricelluar proteins regulating cell growth, differentiation, survival and migration in development, tissue remodeling and repair [49] was prominently up-regulated in metastatic cells. To directly test whether CYR61 played a causal role in the metastatic behavior, we performed gain and loss of function experiments. CYR61 over-expression in low-metastatic variants enhanced lung metastasis formation, while CYR61 silencing in aggressive lines attenuated lung metastasis formation. CYR61 promoted tumor cell survival under hypopxia and Matrigel invasion in vitro [36] (and unpublished observations).

CYR61 was reported to exert many of its biological activities through integrin adhesion receptors $[50,51]$, including $\alpha \vee \beta 3$ and $\alpha \vee \beta 5$, two integrins known to promote invasion and metastasis [52-54]. To test for the functional role of $\alpha \mathrm{V}$ integrins in mediating the pro-invasive and pro-metastatic effects of CYR61, we performed invasion and metastasis experiments in the absence or presence of $\alpha \mathrm{V}$ integrin inhibitors. The function-blocking anti- $\alpha \mathrm{V}$ mAb 17E6 [55] and the $\alpha \vee \beta 3 / \alpha \vee \beta 5$-specific antagonist EMD121974 (Cilengitide) [56] inhibited in vitro Matrigel invasion and lung metastasis formation of tumor cells overexpressing CRY 61 and of tumor cells growing in a pre-irradiate bed. $\alpha \mathrm{V}$ integrin inhibitors impinged on CYR61 mediated survival under hypoxia and enhanced Matrigel invasion in vitro [36] (and unpublished observations).

Taken together, these results demonstrate that CYR61 co-operates with tumor cell $\alpha \mathrm{V}$ integrins to mediate tumor invasion and metastasis (figure 2).

\section{Outstanding questions}

Results originated from these experiments provide a conceptual advance to the understanding of the tumor bed effect. First, they demonstrate that ionizing radiation inhibits angiogenesis by suppressing endothelial sprouting. Second, they show that tumors growing in a bed with suppressed angiogenesis develop evasive resistance, and third, they identify molecular targets to reverse angiogenic blockade (i.e. ALK5) and to suppress metastasis ( $\alpha \mathrm{V}$ integrins). A number of unresolved questions remain, and will need further investigations. Here we will discuss some of them.

\section{Prospective clinical studies addressing the putative metastasis-promoting role of radiotherapy}

While it is clear that tumor or tumor bed irradiation can promote tumor invasion or metastasis of experimental tumors, clinical evidence for this effect in human patients remains controversial. Literature supporting or dismissing such possible effect in patients is based on retrospective analysis of non-randomized studies. Considering the clinical relevance of such an effect, it would be important to design prospective studies explicitly addressing this question in cancer patients treated.

\section{Radiation doses and schedules}

Experimental work in the field of radiotherapy, including our own, is often performed using single high doses of ionizing radiations (e.g. $20 \mathrm{~Gy}$ ). In contrast, clinical radiotherapy involves the repeated delivery of rather low doses of radiation, (i.e. 1.8-2.2 Gy, fractionated therapy). Although single high doses can be calculated to correspond to a clinically-relevant biological cumulative dose of 50-60 Gy (based on the chosen $\alpha / \beta$ values in a linear-quadratic model) [57] delivered to patients during fractionated radiotherapy, in reality we have little knowledge on how endothelial cells react to singles high doses compared to multiple low doses [58]. Considering the clinical relevance of these effects, studies directly comparing the effects on tumor angiogenesis of singles high doses compared to multiple low doses are needed. Experimental evidence indicates that endothelial cell exposure to low single doses (3 Gy) of ionizing radiation promotes endothelial cell survival and capillary formation though activation of Akt signaling [59]. Even lower doses (0.5 Gy) of ionizing radiation can stimulates angiogenesis in vivo resulting in enhanced tumor spreading and metastasis [60]. While high dose ionizing radiation block endothelial cell proliferation and motility, though, at least in part, an ALK5-dependent mechanism, low doses ionizing radiation cause ligand independent VEGF receptor autophosphorylation resulting in increased endothelial cell migration and survival. The reasons for these opposing effects are not clear, but one possibility may relate to differential effects on lipid membranes (causing receptor aggregation and activation) and DNA damage (causing cell cycle arrest and senescence), which are highly dose-dependent. 


\section{Alternative mechanisms of angiogenesis}

While our study clearly shows that sprouting angiogenesis is inhibited by radiation, other mechanisms of angiogenesis may be less affected. For example, Hlushchuk et al. demonstrated that irradiated tumor vessels exposed to angiogenic stimuli switch from sprouting angiogenesis to intussusceptive angiogenesis [61]. Although the resulting microvascular density is reduced by $30 \%$ to $40 \%$ compared to non-irradiated tumors, this alternative form of angiogenesis might be sufficient to support persistence or growth of tumors present in a pre-irradiated bed in spite of fully blocked sprouting angiogenesis. More work is needed to unravel radiation effects on other modes and steps of angiogenesis.

\section{Role of bone marrow derived cells}

While we concentrated our efforts on characterizing direct radiation effects on endothelial cells, it is plausible that within the tumor microenvironment additional radiation-induced effects might play a role in modulating angiogenesis and tumor spreading, including effects on bone marrow-derived cells. Decreased matrix metalloproteinase (MMP)-9 production by $\mathrm{CD} 11 \mathrm{~b}^{+}$bone marrow-derived myelomonocytic cells recruited after irradiation was shown to be responsible for the angiogenic blockade observed in irradiated tumors [62]. Radiation was reported to mobilize bone marowderived vascular progenitors [63] and CD11 b cells, which, once recruited to irradiated tumors, promote vessel formation by vasculogenesis [64]. Furthermore, radiation has been shown to increase recruitment of bone marrow-derived mesenchymal stem cells into tumors via induced alterations in tumor-derived secreted factors [65]. In our model we have observed a $50 \%$ reduction in the number of myelomonocytic cells infiltrating the Matrigel plugs implanted in irradiated mice compared to plugs implanted in control mice. Supplementation of Matrigel plugs with circulating monocytes, however, did not rescue radiation-induced inhibition of angiogenesis, suggesting that their contribution is not essential (N. Imaizumi, unpublished results). In future studies it will be nevertheless important to compare radiation effects on endothelial cells vs. tumor-infiltrating bone marrow-derived cells in deficient angiogenesis and vasculogenic rescue and to determine whether the two mechanisms are somehow connected.

\section{Targeting TGF- $\beta$ and activin receptor-like kinase(ALK) -5 to prevent radiation-induced angiogenic blockade}

The observation that ALK5 inhibition prevented radiation-induced angiogenic blockade [31] suggest the possibility of using an ALK5 kinase inhibitor or an anti-TGF- $\beta$ blocking antibody during radiotherapy to prevent radiation-induced inhibition of angiogenesis and dependent effects. Furthermore, since TGF- $\beta$ can promote epithelial-mesenchymal transition [66], enhanced TGF- $\beta$ signaling might directly contribute to enhance the invasive and metastatic capacities of cancers growing in irradiated beds [67]. While this is an attractive option, great caution should be considered at this point since the TGF- $\beta$ system has pleiotropic activities in normal tissue homeostasis and other pathological conditions and its imbalance may lead to unwanted or unpredictable effects on its own [68]. Inhibitors of ALK5 are in clinical development, including as anti-cancer agents [69] and results generated by these trials will be very informative on this matter.

\section{Combination of radiotherapy and anti-angiogenic therapy}

In 1998 Mauceri et al., reported for the first time that combined administration of radiation therapy and angiostatin-based anti-angiogenic treatment, resulted in increased anti-tumor response without increased toxicity towards normal tissue. These authors showed that radiation and angiostatin had combined cytotoxic effects on endothelial cells, but not tumor cells [70]. Subsequent studies combining radiation therapy with anti-angiogenic [71, 72] or anti-vascular [73] agents, confirmed that concomitant targeting of angiogenic vessels enhanced anti-tumor response to radiotherapy. Further studies investigating the effect of sequential combination of radiotherapy and antiangiogenics demonstrated that anti-angiogenics given after radiotherapy also had a valuable therapeutic effect $[72,74]$. This combination was based on the rationale that radiotherapy induces the expression of angiogenic factors, such as VEGF or FGF-2, which then facilitate tumor re-growth. Combined treatment, however, was reported to increase morbidity [75]. Although initials results appeared to support a combined anti-angiogenic treatment with radiotherapy additional results obtained using different anti-angiogenic agents in different tumor models gave inconsistent results (reviewed in [76]). In 
addition our finding demonstrating that radiotherapy has by itself a direct anti-angiogenic effect, give little rational support for this combination. In short, available experimental results do not consistently support the notion that addition of anti-angiogenic agents to radiotherapy improves efficacy of radiotherapy. In spite of this unresolved issue, however, clinical trials combining anti-angiogenics and radiotherapy have been started and initial results show that is at least well tolerated [77]. Future will tell whether this combination will bring significant survival benefits.

\section{$\alpha \mathbf{V}$ integrin inhibition as a potential therapeutic approach to suppress metastasis}

The fact that $\alpha \mathrm{V}$ integrin inhibition attenuates the invasion and metastasis induced by forced expression of CYR61 and tumor growth in a pre-irradiated bed, might inspire the development of $\alpha \mathrm{V}$ integrin-based therapies for patients at risk for post-radiation recurrences. Importantly, the gene encoding for the integrin $\beta 5$ subunit (ITGB5), one of the $\beta$ subunit associating with $\alpha \mathrm{V}$, was part of the to up-regulated genes that correlated with CYR61 expression. Beyond radiotherapy, a similar approach might be relevant to anti-angiogenic treatments. While patients with advanced cancers clearly benefit from anti-angiogenic treatments [78], tumors eventually escape and progress [48, 79]. Our results suggest that combined administration of $\alpha \mathrm{V}$ integrin antagonists may improve therapeutic efficacy of other existing anti-angiogenic drugs by counteracting hypoxia selection and metastatic dissemination of tumor escape cell variants. Of interest, integrin inhibitors, in particular the arginin, gylcin, aspartic acid-based (RGD) $\alpha \mathrm{V}$ integrin antagonist Cilengitide, are in clinical development as anti-cancer agents [80]. A phase I/Ila study combining Cilengitide and Temozolomide with concomitant radiotherapy followed by cilengitide and temozolomide maintenance therapy in patients with newly diagnosed glioblastoma demonstrated promising activity in patients with MGMT promoter methylation [81]. A phase III study is in progress.

\section{Conclusions and outlook}

Work performed in our laboratory demonstrated that radiation inhibits sprouting angiogenesis through TGF- $\beta / A L K 5$-dependent (i.e. impaired migration and sprouting) and independent (i.e. cell cycle arrest and senescence) mechanisms, and that tumors growing within a pre-irradiated bed become hypoxic, highly invasive and metastatic. We identified the matricellular protein CYR61 and $\alpha \mathrm{V} \beta 5$ integrin as proteins cooperating to mediate invasion and metastasis. An $\alpha \mathrm{V}$ integrin inhibitor in clinical development suppressed metastasis of tumors growing within a pre-irradiated field. Ongoing experiments indicate that bone marrowderived myeolomonocytic cells might also play a significant role in promoting metastasis. These results represent a conceptual advance to the understanding of the tumor bed effect induced by radiotherapy and provide a rationale to develop new therapeutic approaches for preventing tumor escape and metastasis, which can be promptly tested in the clinic. These results might also be relevant to anti-angiogenic therapies, since it was suggested that hypoxia caused by anti-angiogenic treatments may promote tumor progression [82-84] and mathematical modeling indicates that hypoxia drives phenotypic tumor evolution and invasion [85]. Recent experimental results confirmed that anti-angiogenic therapy can drive tumor invasion and metastasis [48]. Combined administration of $\alpha \mathrm{V}$ integrin antagonists, or other anti-invasive drugs (e.g. Met inhibitors) [86] with anti-angiogenic drugs and chemotherapy, may improve clinical outcome of current treatments.

Future research directions include the quest of gene expression signatures predicting tumor resistance and escape from radiotherapy. Such a signature would allow the identification of patients at risk for progression and possibly benefiting from an anti-invasive concomitant therapy early on during treatment. Also, we need to unravel cellular and molecular mechanism mediating invasive growth. We are currently investigating how CYR61 is regulated and how it promotes tumor metastasis. Additional candidate targets have been identified in our screen and are been pursued. Furthermore, dissection of mechanisms of cell survival during hypoxia might deliver important insights into mechanisms of escape. Clinical studies aimed at validating these results in patients should be initiated and conducted in association with laboratory investigations.

Acknowledgments. Work in our laboratory is supported by grants from the Swiss National Science Foundation (SNF), the National Center for Competence in Research (NCCR, molecular Oncology, a research instrument of the SNF), Swiss Cancer League and Oncosuisse, the CCRP program from Oncosuisse, the Medic Foundation, and the 7 th Frame program from the European Union. We thank 
S. Goodman, M. Hegi, R. Stupp, R.O. Mirimanoff for helpfully discussions and P. Farmer, T. Sengstag and M. Delorenzi for bioinfomatic analysis, J.C. Stehle, S. Andrejevic-Blant, G. Bieler and R. Moeckli for help with the experiments.

Conflict of interest : none.

\section{References}

1. Bernier J, Hall EJ, Giaccia A. Radiation oncology: a century of achievements. Nat Rev Cancer 2004; 4:737-47.

2. Gudkov AV, Komarova EA. The role of p53 in determining sensitivity to radiotherapy. Nat Rev Cancer $2003 ; 3: 117-29$.

3. Barcellos-Hoff MH, Park C, Wright EG. Radiation and the microenvironment - tumorigenesis and therapy. Nat Rev Cancer $2005 ; 5: 867-75$

4. Garcia-Barros M, Paris F, Cordon-Cardo C, et al. Tumor response to radiotherapy regulated by endothelial cell apoptosis. Science $2003 ; 300: 1155-9$.

5. Bartelink $\mathrm{H}$, Horiot JC, Poortmans $\mathrm{P}$, et al. Recurrence rates after treatment of breast cancer with standard radiotherapy with or without additional radiation. N Engl J Med 2001 ; 345 : 1378-87.

6. Vicini FA, Kestin L, Huang R, Martinez A. Does local recurrence affect the rate of distant metastases and survival in patients with early-stage breast carcinoma treated with breast-conserving therapy? Cancer $2003 ; 97: 910-9$.

7. Vikram B, Strong EW, Shah JP, Spiro R. Failure at distant sites following multimodality treatment for advanced head and neck cancer. Head Neck Surg $1984 ; 6: 730-3$.

8. Suit HD. Local control patient survival. Int / Radiat Oncol Biol Phys $1992 ; 23: 653-60$

9. O'Brien CJ, Smith JW, Soong SJ, Urist MM, Maddox WA. Neck dissection with and without radiotherapy: prognostic factors, patterns of recurrence and survival. Am / Surg 1986; 152:456-63.

10. Milas L, Hirata $\mathrm{H}$, Hunter $\mathrm{N}$, Peters LJ. Effect of radiation-induced injury of tumor bed stroma on metastatic spread of murine sarcomas and carcinomas. Cancer Res $1988 ; 48: 2116-20$.

11. Milas L, Hunter N, Peters LJ. The tumor bed effect: dependence of tumor take, growth rate, and metastasis on the time interval between irradiation and tumor cell transplantation. Int I Radiat Oncol Biol Phys $1987 ; 13: 379-83$

12. Woodward WA, Truong PT, Yu TK, et al. Among women who experience a recurrence after postmastectomy radiation therapy irradiation is not associated with more aggressive local recurrence or reduced survival. Breast Cancer Res Treat 2010 ; 123 : 597-605.

13. Rofstad EK. Microenvironment-induced cancer metastasis. Int $)$ Radiat Biol $2000 ; 76: 589-605$.

14. Rofstad EK, Mathiesen B, Henriksen K, Kindem K, Galappathi $K$. The tumor bed effect: increased metastatic dissemination from hypoxia-induced up-regulation of metastasis-promoting gene products. Cancer Res $2005 ; 65: 2387-96$.

15. Kozin SV, Winkler F, Garkavtsev I, Hicklin DJ, Jain RK, Boucher $Y$. Human tumor xenografts recurring after radiotherapy are more sensitive to anti-vascular endothelial growth factor receptor-2 treatment than treatment-naive tumors. Cancer Res 2007; 67:5076-82.

16. Bonnaud S, Niaudet C, Pottier G, et al. Sphingosine-1-phosphate protects proliferating endothelial cells from ceramide-induced apoptosis but not from DNA damage-induced mitotic death. Cancer Res $2007 ; 67: 1803-11$

17. Nieder C, Wiedenmann N, Andratschke NH, Astner ST, Molls M. Radiation therapy plus angiogenesis inhibition with bevacizumab: rationale and initial experience. Rev Recent Clin Trials 2007;2: 163-8.
18. Fenton BM, Paoni SF. The addition of AG-013736 to fractionated radiation improves tumor response without functionally normalizing the tumor vasculature. Cancer Res 2007; 67 : 9921-8.

19. Shinohara ET, Cao C, Niermann K, et al. Enhanced radiation damage of tumor vasculature by mTOR inhibitors. Oncogene $2005 ; 24: 5414-22$.

20. Wachsberger P, Burd R, Dicker AP. Improving tumor response to radiotherapy by targeting angiogenesis signaling pathways. Hematol Oncol Clin North Am $2004 ; 18: 1039-57$.

21. Fajardo LF. The pathology of ionizing radiation as defined by morphologic patterns. Acta Oncol $2005 ; 44$ : 13-22.

22. Tetik O, Yetkin U, Calli AO, Ilhan G, Gurbuz A. Occlusive arterial disease after radiotherapy for testicular cancer: case report and review of the literature. Vascular $2008 ; 16: 239-41$.

23. Group EBCTC. Favourable and unfavourable effects on long-term survival of radiotherapy for early breast cancer: an overview of the randomised trials. Lancet $2000 ; 355: 1757-70$.

24. Dorresteijn LD, Kappelle AC, Scholz NM, et al. Increased carotid wall thickening after radiotherapy on the neck. Eur / Cancer $2005 ; 41: 1026-30$

25. Little MP, Tawn EJ, Tzoulaki I, et al. A systematic review of epidemiological associations between low and moderate doses of ionizing radiation and late cardiovascular effects, and their possible mechanisms. Radiat Res 2008; 169:99-109.

26. Potten CS. A comprehensive study of the radiobiological response of the murine (BDF1) small intestine. Int / Radiat Biol $1990 ; 58: 925-73$

27. Paris F, Fuks Z, Kang A, et al. Endothelial apoptosis as the primary lesion initiating intestinal radiation damage in mice. Science $2001 ; 293$ : 293-7.

28. Maj JG, Paris F, Haimovitz-Friedman A, Venkatraman E, Kolesnick R, Fuks Z. Microvascular function regulates intestinal crypt response to radiation. Cancer Res $2003 ; 63: 4338-41$.

29. Kirsch DG, Santiago PM, di Tomaso E, et al. p53 controls radiation-induced gastrointestinal syndrome in mice independent of apoptosis. Science 2010; 327:593-6.

30. Dormand EL, Banwell PE, Goodacre TE. Radiotherapy wound healing. Int Wound / $2005 ; 2$ :112-27.

31. Imaizumi $I$, Monnier $Y$, Hegi $M$, Mirimanoff RO, Rüegg C. Radiotherapy suppresses angiogenesis in mice through TGFBRI/ALK5-dependent inhibition of endothelial cell sprouting. PLOSONE 2010 [in press].

32. Fei P, El-Deiry WS. P53 and radiation responses. Oncogene $2003 ; 22: 5774-83$

33. Barcellos-Hoff MH, Derynck R, Tsang ML, Weatherbee JA. Transforming growth factor-beta activation in irradiated murine mammary gland. J Clin Invest 1994; 93 : 892-9.

34. Randall K, Coggle JE. Expression of transforming growth factorbeta 1 in mouse skin during the acute phase of radiation damage. Int I Radiat Biol 1995; 68:301-9.

35. Barcellos-Hoff MH, Costes SV. A systems biology approach to multicellular and multi-generational radiation responses. Mutat Res $2006 ; 597: 32-8$.

36. Monnier $\mathrm{Y}$, Farmer $\mathrm{P}$, Bieler $\mathrm{G}$, et al. CYR61 and alphaVbeta5 integrin cooperate to promote invasion and metastasis of tumors growing in preirradiated stroma. Cancer Res 2008;68: 7323-31.

37. Leith JT, Padfield G, Quaranto L, Michelson S. Effect of preirradiation of transplantation site on growth characteristics and hypoxic fractions in human colon tumor xenografts. Cancer Res $1992 ; 52: 2162-6$

38. Penhaligon M, Courtenay VD, Camplejohn RS. Tumour bed effect: hypoxic fraction of tumours growing in preirradiated beds. Int / Radiat Biol Relat Stud Phys Chem Med 1987;52: 635-41. 
39. Hockel M, Schlenger K, Hockel S, Aral B, Schaffer U, Vaupel P. Tumor hypoxia in pelvic recurrences of cervical cancer. Int I Cancer $1998 ; 79: 365-9$.

40. Cairns RA, Kalliomaki T, Hill RP. Acute (cyclic) hypoxia enhances spontaneous metastasis of KHT murine tumors. Cancer Res $2001 ; 61: 8903-8$

41. Rofstad EK, Halsor EF. Hypoxia-associated spontaneous pulmonary metastasis in human melanoma xenografts: involvement of microvascular hot spots induced in hypoxic foci by interleukin $8 . \mathrm{Br}$ J Cancer $2002 ; 86: 301-8$.

42. Brizel DM, Dodge RK, Clough RW, Dewhirst MW. Oxygenation of head and neck cancer: changes during radiotherapy and impact on treatment outcome. Radiother Oncol 1999; 53 : 113-7.

43. Hockel M, Schlenger K, Aral B, Mitze M, Schaffer U, Vaupel P. Association between tumor hypoxia and malignant progression in advanced cancer of the uterine cervix. Cancer Res 1996; 56:450915

44. Brizel DM, Scully SP, Harrelson JM, et al. Tumor oxygenation predicts for the likelihood of distant metastases in human soft tissue sarcoma. Cancer Res 1996; 56:941-3.

45. Harris AL. Hypoxia-a key regulatory factor in tumour growth. Nat Rev Cancer $2002 ; 2: 38-47$

46. Pouyssegur J, Dayan F, Mazure NM. Hypoxia signalling in cancer and approaches to enforce tumour regression. Nature $2006 ; 441: 437-43$

47. Gassmann M, Kvietikova I, Rolfs A, Wenger RH. Oxygen- and dioxin-regulated gene expression in mouse hepatoma cells. Kidney Int 1997;51:567-74.

48. Paez-Ribes M, Allen E, Hudock J, et al. Antiangiogenic therapy elicits malignant progression of tumors to increased local invasion and distant metastasis. Cancer Cell 2009; 15:220-31.

49. Leask A, Abraham DJ. All in the CCN family: essential matricellular signaling modulators emerge from the bunker. / Cell SCi $2006 ; 119: 4803-10$

50. Lau LF, Lam SC. The CCN family of angiogenic regulators: the integrin connection. Exp Cell Res 1999 ; 248:44-57.

51. Menendez JA, Mehmi I, Griggs DW, Lupu R. The angiogenic factor CYR61 in breast cancer: molecular pathology and therapeutic perspectives. Endocr Relat Cancer 2003 ; 10:141-52.

52. Brooks PC, Klemke RL, Schon S, Lewis JM, Schwartz MA, Cheresh DA. Insulin-like growth factor receptor cooperates with integrin alpha $\checkmark$ beta 5 to promote tumor cell dissemination in vivo. J Clin Invest $1997 ; 99: 1390-8$

53. Enns A, Korb T, Schluter K, et al. Alphavbeta5-integrins mediate early steps of metastasis formation. Eur J Cancer $2005 ; 41$ : 1065-72.

54. Felding-Habermann B. Integrin adhesion receptors in tumor metastasis. Clin Exp Metastasis $2003 ; 20: 203-13$.

55. Mitjans F, Sander D, Adan J, et al. An anti-alpha v-integrin antibody that blocks integrin function inhibits the development of a human melanoma in nude mice. / Cell Sci 1995; 108:2825-38.

56. Dechantsreiter MA, Planker E, Matha B, et al. N-Methylated cyclic RGD peptides as highly active and selective alpha(V) beta(3) integrin antagonists. J Med Chem 1999; 42 :3033-40.

57. Barton M. Tables of equivalent dose in 2 Gy fractions: a simple application of the linear quadratic formula. Int I Radiat Oncol Biol Phys $1995 ; 31: 371-8$

58. Brown JM, Koong AC. High-dose single-fraction radiotherapy: exploiting a new biology? Int / Radiat Oncol Biol Phys 2008; $71: 324$ 5.

59. Tan I, Geng L, Yazlovitskaya EM, Hallahan DE. Protein kinase B/Akt-dependent phosphorylation of glycogen synthase kinase-3beta in irradiated vascular endothelium. Cancer Res 2006 ; 66 :2320-7.

60. Sofia Vala I, Martins LR, Imaizumi N, et al. Low doses of ionizing radiation promote tumor growth and metastasis by enhancing angiogenesis. PLOSONE 2010; 5 : e11222.
61. Hlushchuk R, Riesterer $\mathrm{O}$, Baum $\mathrm{O}$, et al. Tumor recovery by angiogenic switch from sprouting to intussusceptive angiogenesis after treatment with PTK787/ZK222584 or ionizing radiation. Am J Pathol 2008; $173: 1173-85$

62. Ahn GO, Brown JM. Matrix metalloproteinase-9 is required for tumor vasculogenesis but not for angiogenesis: role of bone marrowderived myelomonocytic cells. Cancer Cell 2008; 13 : 193-205.

63. Allan DS, Morgan SC, Birch PE, et al. Mobilization of circulating vascular progenitors in cancer patients receiving external beam radiation in response to tissue injury. Int / Radiat Oncol Biol Phys $2009 ; 75: 220-4$.

64. Kioi M, Vogel H, Schultz G, Hoffman RM, Harsh GR, Brown JM. Inhibition of vasculogenesis, but not angiogenesis, prevents the recurrence of glioblastoma after irradiation in mice. $J$ Clin Invest $2010 ; 120: 694-705$.

65. Klopp AH, Spaeth EL, Dembinski JL, et al. Tumor irradiation increases the recruitment of circulating mesenchymal stem cells into the tumor microenvironment. Cancer Res 2007; 67 : 11687-95.

66. Jakowlew SB. Transforming growth factor-beta in cancer and metastasis. Cancer Metastasis Rev 2006 ; 25 : 435-57.

67. Park CC, Bissell MJ, Barcellos-Hoff $\mathrm{MH}$. The influence of the microenvironment on the malignant phenotype. Mol Med Today $2000 ; 6: 324-9$

68. Kim IY, Kim MM, Kim SJ. Transforming growth factor-beta: biology and clinical relevance. J Biochem Mol Biol 2005 ; 38 : 1-8.

69. Lahn M, Kloeker S, Berry BS. TGF-beta inhibitors for the treatment of cancer. Expert Opin Investig Drugs 2005; 14 : 629-43.

70. Mauceri HJ, Hanna NN, Beckett MA, et al. Combined effects of angiostatin and ionizing radiation in antitumour therapy. Nature $1998 ; 394: 287-91$

71. Ning S, Laird D, Cherrington JM, Knox SJ. The antiangiogenic agents SU5416 and SU6668 increase the antitumor effects of fractionated irradiation. Radiat Res $2002 ; 157: 45-51$.

72. Schueneman AJ, Himmelfarb E, Geng L, et al. SU11248 maintenance therapy prevents tumor regrowth after fractionated irradiation of murine tumor models. Cancer Res $2003 ; 63: 4009-16$.

73. Hoang T, Huang S, Armstrong E, EickhoffJC, Harari PM. Augmentation of radiation response with the vascular targeting agent ZD6126. Int J Radiat Oncol Biol Phys 2006; 64 : 1458-65.

74. Amano M, Suzuki M, Andoh S, et al. Antiangiogenesis therapy using a novel angiogenesis inhibitor, anginex, following radiation causes tumor growth delay. Int J Clin Oncol 2007; $12: 42-7$.

75. Verhoeff JJ, Stalpers LJ, Van Noorden CJ, et al. Angiogenesis inhibitor DC101 delays growth of intracerebral glioblastoma but induces morbidity when combined with irradiation. Cancer Lett $2009 ; 285: 39-45$

76. Classen J, Budach W. Antiangiogenesis and radiotherapy: what is the role of combined modality treatment? Curr Med Chem Anticancer Agents $2003 ; 3: 375-82$.

77. Gutin PH, Iwamoto FM, Beal K, et al. Safety and efficacy of bevacizumab with hypofractionated stereotactic irradiation for recurrent malignant gliomas. Int / Radiat Oncol Biol Phys 2009; 75 : 156-63.

78. Gasparini G, Longo R, Toi M, Ferrara N. Angiogenic inhibitors: a new therapeutic strategy in oncology. Nat Clin Pract Oncol $2005 ; 2: 562-77$.

79. Casanovas O, Hicklin DJ, Bergers G, Hanahan D. Drug resistance by evasion of antiangiogenic targeting of VEGF signaling in late-stage pancreatic islet tumors. Cancer Cell $2005 ; 8$ : 299-309.

80. Stupp R, Ruegg C. Integrin inhibitors reaching the clinic. J Clin Oncol $2007 ; 25: 1637-8$

81. Stupp R, Hegi ME, Neyns B, et al. Phase I/Ila Study of cilengitide and temozolomide with concomitant radiotherapy followed by cilengitide and temozolomide maintenance therapy in patients with newly diagnosed glioblastoma. J Clin Oncol 2010;28 :2712-8. 
82. Steeg PS. Angiogenesis inhibitors: motivators of metastasis? Nat Med $2003 ; 9: 822-3$.

83. Bottaro DP, Liotta LA. Cancer: out of air is not out of action. Nature $2003 ; 423: 593-5$.

84. Blagosklonny MV. Antiangiogenic therapy tumor progression. Cancer Cell $2004 ; 5: 13-7$.
85. Anderson AR, Weaver AM, Cummings PT, Quaranta V. Tumor morphology and phenotypic evolution driven by selective pressure from the microenvironment. Cell 2006;127:905-15.

86. Mazzone M, Comoglio PM. The Met pathway: master switch and drug target in cancer progression. FASEB / 2006;20: 1611-21. 